\title{
Diversity and frequency of $k d r$ mutations within Anopheles sinensis populations from Guangxi, China
}

\author{
Chan Yang ${ }^{1,3+}{ }^{+}$Xiangyang Feng ${ }^{2 \dagger}$, Zushi Huang ${ }^{1}$, Mei Li ${ }^{1}$ and Xinghui Qiu ${ }^{1,3 *}$
}

\begin{abstract}
Background: Anopheles sinensis is a major vector of malaria in China and its control is under great threat as the development of insecticide resistance. Voltage-gated sodium channel (VGSC) is the target of several classes of insecticides. Genetic mutations of VGSC have been documented to confer knockdown resistance (kdr) to dichlorodiphenyltrichloroethane (DDT) and pyrethroids in mosquitoes. To control this vector efficiently, it is important to know the resistanceassociated genetic mutations, their distribution frequencies and genealogical relations.

Methods: Three hundreds and thirteen (313) adults of An. sinensis collected from nine locations across Guangxi Zhuang Autonomous Region were used. The partial sequence of the An. sinensis voltage gated sodium channel gene (AS-VGSC) containing codon 1014 was sequenced. PHASE2.1 was used to construct the haplotypes of each individual, and the accuracy of haplotypes was further confirmed by clone sequencing. The genealogical relations of $k d r$ mutations in AS-VGSC was analysed using TCS 2.1 and Network 5.0.

Results: Sixteen AS-VGSC haplotypes including seven haplotypes carrying non-synonymous mutations at codon 1014, and fifty-five AS-VGSC genotypes were identified from 313 mosquitoes collected from nine geographical locations across Guangxi. The number of haplotypes in each of the nine populations ranged from 5 to 13. The frequency of haplotypes carrying $k d r$ mutations ranged from 2.7 to $80.0 \%$ within the nine populations, of which 1014C was unexpectedly high in the northeast of Guangxi. Genealogical analysis suggested multiple origins of kdr mutations in An. sinensis.

Conclusion: Diverse haplotypes of AS-VGSC are distributed in Guangxi. The presence of haplotypes carrying mutations at codon 1014 indicates a risk of pyrethroid and DDT resistance. The kdr mutations show differential distribution geographically, with high frequencies occurred in the northeast of Guangxi. Genealogical analysis suggests multiple origins of kdr mutations in An. sinensis populations in Guangxi. These findings have important practical implications for the sustainability of An. sinensis control programmes.
\end{abstract}

Keywords: Anopheles sinensis, Voltage-gated sodium channel (VGSC), Knockdown resistance (kdr), Haplotype, Genealogical analysis, Guangxi Zhuang Autonomous Region

\section{Background}

Anopheles sinensis is a major vector of malaria in China and countries of Southeast Asia. Chemical control of

\footnotetext{
*Correspondence: qiuxh@ioz.ac.cn

${ }^{\dagger}$ Chan Yang and Xiangyang Feng contributed equally to this work.

${ }^{1}$ State Key Laboratory of Integrated Management of Pest Insects and Rodents, Institute of Zoology, Chinese Academy of Sciences, Beijing 100101, China

Full list of author information is available at the end of the article
}

vector has played an important role in malaria control and elimination [1]. In China, DDT has been widely used for indoor residual spray (IRS) since 1950s, and pyrethroids for insecticide-treated bed nets (ITNs) since 1980s [2]. Unfortunately, long-term and large-scale use of pyrethroids has led to increasing insecticide resistance in Chinese An. sinensis [3, 4], which poses a major threat to malaria control.

\section{Biomed Central}

(c) 2016 The Author(s). This article is distributed under the terms of the Creative Commons Attribution 4.0 International License (http://creativecommons.org/licenses/by/4.0/), which permits unrestricted use, distribution, and reproduction in any medium, provided you give appropriate credit to the original author(s) and the source, provide a link to the Creative Commons license, and indicate if changes were made. The Creative Commons Public Domain Dedication waiver (http://creativecommons.org/ publicdomain/zero/1.0/) applies to the data made available in this article, unless otherwise stated. 
The voltage-gated sodium channel protein is the major target for pyrethroids and DDT [5]. Although there have been debates [6-8], many studies have demonstrated that mutations at codon 1014 of $V G S C$ cause resistance to both pyrethroids and DDT in many arthropod species $[5,6] .1014 \mathrm{~F} / \mathrm{S} / \mathrm{H}$ mutations can reduce sodium channel sensitivity to pyrethroids in Xenopus oocytes [9-15], and provide protection to pyrethroids and DDT [9]. In $A n$. sinensis, significant positive correlations between $k d r$ allele frequency and bioassay-based resistance phenotype have been documented [16-19]. Recent years, $k d r$ mutation has been used as a molecular mark for monitoring pyrethroid resistance in An. sinensis in China [19].

Guangxi Zhuang Autonomous Region was once a malaria-endemic region. Before 1949, there were more than 5 million malaria patients per year [20]. Thanks to the "National Malaria Control Programme", the "Basically Eliminating Malaria" strategy and the "Action Plan of Malaria Elimination (2010-2020)" implemented since 1955, 2000, and 2010 respectively in China [21, 22], the malaria burden has been substantially reduced, with one indigenous and 2068 imported cases being reported from 2010 to 2015 in Guangxi [22]. Although Guangxi has already achieved remarkable accomplishments in eliminating malaria, the risk of malaria re-emergence remains partly due to increasing cross-border population migration and the unique natural environment (e.g. rice fields) suitable for mosquito breeding in Guangxi [22-25].

As the historical use in vector control and continuing use in agriculture of different insecticides, not only the geographical distribution and density of malaria vectors are likely to change, insecticide resistance is expected to be selected as well. Recent investigations have shown that An. sinensis has replaced Anopheles minimus to be the main malaria vector in Guangxi [24]. However, the status of insecticide resistance in $A n$. sinensis is less understood in this region up to date. As an effort in this direction, the distribution and frequency of VGSC mutations that possibly lead to resistance to DDT and pyrethroids were investigated in nine field populations of An. sinensis collected extensively across Guangxi in this study.

\section{Methods}

\section{Samples}

Anopheles sinensis adults used in the study were caught by light trap (wave length $365 \mathrm{~nm}$ ) from July to September in 2014, around farmers' houses at different geographical locations across Guangxi. The sampling sites were part of the malaria vector monitoring sites that were set up partly because there were imported malaria cases observed since 2010 in these regions. A brief description of the sampling sites was provided in Additional file 1 . The farmers living at the sampling sites usually use mosquito-coil (largely containing pyrethroids) to prevent mosquitoes, and apply insecticides (e.g. pyrethroids) for crop protection.

The collected individual mosquitoes were first morphologically identified using the determination key provided by [26]. Anopheles sinensis adults were put into $100-\mu \mathrm{l}$ Eppendorf tubes containing $100 \%$ ethanol solution, and kept at $4{ }^{\circ} \mathrm{C}$ until use. The accuracy of morphological classification was guaranteed by molecular detection of ten randomly selected adults from each population using the rDNA-ITS2 method [27].

\section{PCR amplification of partial AS-VGSC sequence}

The genomic DNA of individual adult mosquitoes was prepared as described elsewhere [28, 29]. A fragment containing codon 1014 of $A S-V G S C$ gene was amplified using primers $\mathrm{kdr}-\mathrm{F}$ (5'-TGCCACTCCGT GTGTTTAGA-3') and $k d r$-R (5'-GAGCGATGAT GATCCGAAAT- $\left.{ }^{\prime}\right)$ [16]. The PCR mixture $(30 \mu \mathrm{l})$ consisted of $6 \mu$ l DNA template, $0.4 \mu$ l Takara Taq Polymerase, $3 \mu \mathrm{l}$ 10xTaq Buffer, $3 \mu \mathrm{l} 2.5 \mu \mathrm{M}$ dNTPs, $0.6 \mu \mathrm{l}$

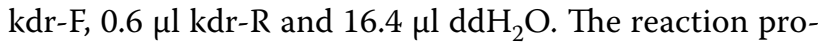
gramme was $95^{\circ} \mathrm{C}$ for $5 \mathrm{~min}$, followed by 36 cycles each with $95{ }^{\circ} \mathrm{C}$ for $30 \mathrm{~s}, 55^{\circ} \mathrm{C}$ for $30 \mathrm{~s}, 72{ }^{\circ} \mathrm{C}$ for $30 \mathrm{~s}$, and by a final extension of $10 \mathrm{~min}$ at $72{ }^{\circ} \mathrm{C}$. PCR products were gel-purified and sequenced using the forward primer $k d r-\mathrm{F}$ (BGI, China).

\section{Haplotype identification and genealogical analysis} Genotype data were carefully inspected by using Chromas (Technelysium Pty Ltd, Australia). AS-VGSC haplotypes were constructed by software PHASE2.1 (http:// www.bioinf.man.ac.uk/resources/phase/) from the genotype data. The presence (and accuracy) of haplotypes constructed in heterozygotes was confirmed by sequencing of three to five clones derived from 12 representative heterozygotes. Genealogical relations among haplotypes were analysed using Network 5.0 [30] and TCS2.1 [31].

\section{Results}

Identification of polymorphic sites in the AS-VGSC gene The gene of $A n$. sinensis voltage sensitive sodium channel (KE525266.1)contains 31 exons and 30 introns. A $~ 325$ bp-in-length sequence was sequenced through direct sequencing of each purified PCR product. The partial sequence $(267 \mathrm{bp})$, excluding the primer sequence and covering partial exon 19 (169 bp), intron 19 (64 bp) and partial exon 20 (34 bp), was used for further analysis (Fig. 1). Two synonymous mutations (C/G at PS1, T/C at PS9) were observed. Five polymorphic sites were found in the intron 19 (PS4-8). Non-synonymous mutations were identified in the second $(\mathrm{T} / \mathrm{G} / \mathrm{C})$ and third nucleotide (G/T) of codon 1014 (PS2 and 3). 


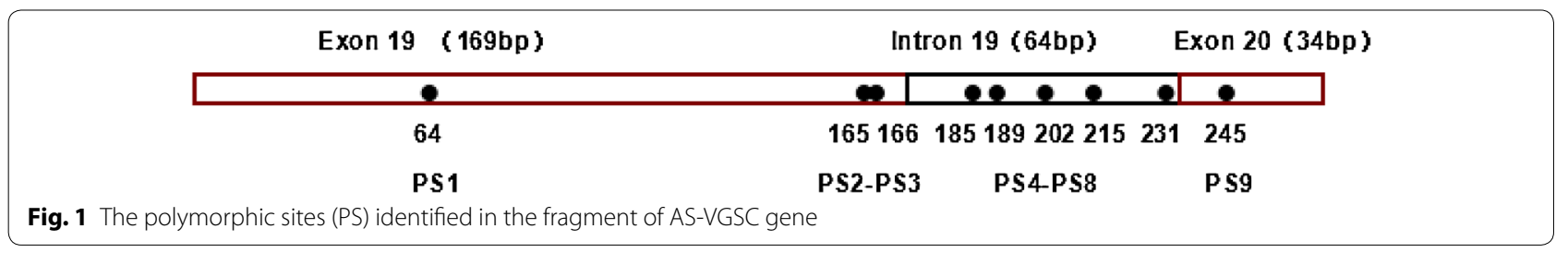

\section{Haplotype diversity of AS-VGSC gene}

From the sequence data of 313 individuals, sixteen $A S$ $V G S C$ haplotypes were identified (Table 1). The number of haplotypes in each of the nine populations ranged from 5 (Guigang) to 13 (Yulin). Three haplotypes (1014L2, 1014L3 and 1014L8) were detected in all the nine populations, while 1014L7 was uniquely distributed in Nanning. 1014L1 was the predominant haplotype for Nanning and Yulin, 1014L2 for Baise and Hechi, and 1014L8 was most abundant in Wuzhou (Table 2).

Point mutations at position 1014 of the domain II of the VGSC protein have been documented to confer $k d r$ [5, 6]. In this study, three types of $k d r$ mutations were detected: TTT (1014F), TCG (1014S), and TGT (1014C). Accordingly, seven haplotypes carrying $k d r$ mutations, namely 1014F1, 1014F2, 1014S1, 1014S2, 1014S3, 1014S4 and $1014 \mathrm{C} 1$ respectively were identified (Table 1 ), in the frequencies ranging from 2.7 to $80.0 \%$ in the nine populations (Fig. 2). In the four populations in the northeast (Guigang, Guilin, Hezhou and Liuzhou), the frequencies of $k d r$ mutations were more than $50.0 \%$, predominated by $1014 \mathrm{C} 1$. In contrast, only $1014 \mathrm{~S}$ in a low frequency $(<5 \%)$ was observed in the two western populations (Baise and Nanning). Three types of $k d r$ mutations coexisted in the other three populations (Hechi, Wuzhou, Yilin) in a frequency from 11.6 to $33.3 \%$.

Fifty-five $A S$-VGSC genotypes were detected (Additional file 2). Among the nine populations, the predominant genotype was largely population-specific. According to the encoding amino acid at position 1014, all the 55 genotypes could be grouped into wild-type (1014L/1014L), mutant-type (1014F/1014F, 1014C/1014C and 1014F/1014C), heterozygous-type (1014L/1014S, 1014L/1014C and 1014L/1014F) (Table 3). Wild-type was found in all the nine populations, in frequencies ranging from 5.3 to $94.6 \%$. No mutant-type individual was observed in Baise, Hechi, Nanning and Yulin. The mutant homozygote $1014 \mathrm{~F} / 1014 \mathrm{~F}$ was only observed in Hezhou in a low frequency of $3.7 \%$, while $1014 \mathrm{C} / 1014 \mathrm{C}$ homozygote was detected in Guigang (60.0\%), Hezhou (42.1\%), Liuzhou (22.3 \%), Guilin (15.8\%) and Wuzhou (8.3\%).

\section{Genealogical analysis of $k d r$ mutations}

To estimate the evolutionary relationship of $k d r$ mutations, sixteen haplotypes identified in this study and ten haplotypes retrieved from GenBank database (Table 1) were used to construct a network using TCS 2.1 and Network 5.0. The two software generated identical results for our dataset, thus only the network produced by Network 5.0 was displayed (Fig. 3).

The network analysis revealed complex reticulate pattern and multiple independent mutation events leading to $k d r$ haplotypes. For example, haplotype 1014F1 was possibly derived from 1014L1, while 1014F2 from 1014L3; 1014S1, S2, S3 and S4 were perhaps the results of an independent mutational step from four different wild haplotypes 1014L5, 1014L2, 1014L3 and 1014L4 respectively.

The genealogical analysis also revealed that a single mutation (T165C) might result in the resistant phenotype from the susceptible one. For example, 1014S2 shared identical intron sequence with 1014L2, L5 and S1, and a single mutation (T165C) might change the susceptible 1014L2 to the resistant 1014S2.

\section{Discussion}

Sequence analysis reveals that the sodium channel gene of $A n$. sinensis has diverse genetic mutations. From 313 mosquitoes collected from Guangxi, 16 haplotypes were identified (Tables 1, 2). The number and frequency of $A S$-VGSC haplotypes are different among the nine populations from Guangxi. Overall, the 1014L1, 1014L2 and $1014 \mathrm{C} 1$ rank the top three haplotypes. Some haplotypes, for example 1014L2, 1014L3 and 1014L8, are widespread, and some haplotypes such as 1014L7 are restricted to a particular location (Table 2). Less number of haplotypes detected in Hezhou and Guigang may be related to the relative small sample size and/or strong selective pressure leading to $1014 \mathrm{C} 1$ in a high frequency. The high polymorphism of $A S-V G S C$ may be partly explained by their large population size, wide distribution range of $A n$. sinensis [14], diverse natural landscapes and different local insecticide selective pressure.

Three non-synonymous mutations (TTT for 1014F, TGT for $1014 \mathrm{C}$ and TCG for 1014S) were identified in this work. However, the TTC-encoding 1014F, previously found in Korea [32] and in Anhui and Sichuan of China [19], was not detected in Guangxi. The presence of L1014W substitution was reported based on the direct sequencing data of only one $A n$. 
Table 1 Haplotypes identified or/and used in this study

\begin{tabular}{|c|c|c|c|}
\hline Haplotype & Polymorphic sites & Intron type & Known distribution in China (Province) $^{a}$ \\
\hline 1014L1 & CTGACGTTC & $\mathrm{H} 1$ & $\begin{array}{l}\text { Fujian, Guangxi, Guangdong, Guizhou, Hainan, Henan, Sichuan, } \\
\text { Yunnan }\end{array}$ \\
\hline $1014 \mathrm{~L} 2$ & CTGACGCCT & $\mathrm{H} 2$ & $\begin{array}{l}\text { Fujian, Guangxi, Guangdong, Guizhou, Hainan, Henan, Sichuan, } \\
\text { Yunnan }\end{array}$ \\
\hline $1014 \mathrm{~L} 3$ & CTGACTCCT & $\mathrm{H} 3$ & Fujian, Guangxi, Guizhou, Hainan, Henan, Sichuan, Yunnan \\
\hline $1014 L 4$ & CTGACTTCT & $\mathrm{H} 4$ & Fujian, Guangxi, Hainan, Yunan \\
\hline $1014 L 5$ & GTGACGCCT & $\mathrm{H} 2$ & Fujian, Guangxi, Guangdong, Hainan, Sichuan \\
\hline $1014 L 6$ & CTGTCGCCT & H5 & Guangxi, Hainan, Sichuan, Yunnan \\
\hline 1014L7 & CTGACGTCC & $\mathrm{H} 6$ & Guangxi, Yunnan \\
\hline 1014L8 & CTGATGCCT & $\mathrm{H} 7$ & Guangxi, Hainan \\
\hline $1014 L 9$ & CTGACTTCC & $\mathrm{H} 4$ & Guangxi \\
\hline $1014 \mathrm{~L} 10^{\mathrm{b}}$ & CTGACGTCT & $\mathrm{H} 6$ & Guizhou, Shandong \\
\hline $1014 \mathrm{~L} 11^{\mathrm{b}}$ & CTGACTTTC & $\mathrm{H} 8$ & Hainan \\
\hline $1014 \mathrm{~L} 12^{\mathrm{b}}$ & CTGACTCCC & $\mathrm{H} 3$ & Sichuan \\
\hline $1014 \mathrm{~L} 13^{\mathrm{b}}$ & CTGTCGCCC & $\mathrm{H} 5$ & Yunnan \\
\hline $1014 \mathrm{~L} 14^{\mathrm{b}}$ & CTGACGCTT & $\mathrm{H} 9$ & Anhui \\
\hline $1014 \mathrm{~F} 1$ & CTTACGTTC & $\mathrm{H} 1$ & $\begin{array}{l}\text { Anhui, Guangxi, Guizhou,Henan, Hubei, } \\
\text { Jiangsu, Shandong }\end{array}$ \\
\hline $1014 F 2$ & CTTACTCCT & $\mathrm{H} 3$ & Anhui, Guangxi, Hainan, Henan, Hubei, Jiangsu \\
\hline $1014 \mathrm{~F} 3^{\mathrm{b}}$ & CTCACTCCT & $\mathrm{H} 3$ & Hubei, Jiangsu, Shandong \\
\hline $1014 \mathrm{~F} 4^{\mathrm{b}}$ & CTTACTTTC & $\mathrm{H} 8$ & Anhui \\
\hline $1014 \mathrm{~F} 5^{\mathrm{b}}$ & CTCACGTCC & $\mathrm{H} 6$ & Henan \\
\hline $1014 \mathrm{FG}^{\mathrm{b}}$ & CTTACGCCT & $\mathrm{H} 2$ & Henan \\
\hline $1014 S 1$ & GCGACGCCT & $\mathrm{H} 2$ & Anhui, Guangxi, Guangdong, Henan \\
\hline $1014 S 2$ & CCGACGCCT & $\mathrm{H} 2$ & Guangxi, Guangdong \\
\hline $1014 S 3$ & CCGACTCCT & $\mathrm{H} 3$ & Guangxi \\
\hline 101454 & CCGACTTCT & $\mathrm{H} 4$ & Guangxi \\
\hline $1014 \mathrm{C} 1$ & CGTACGTTC & $\mathrm{H} 1$ & Anhui, Guangxi, Guizhou, Hubei, Jiangsu \\
\hline $1014 C 2^{b}$ & CGTACTTTC & $\mathrm{H} 8$ & Anhui \\
\hline
\end{tabular}

a Distribution information is adopted from cited references and this study. The polymorphic sites within intron 19 are in italics

b Mean that these haplotypes are retrieved from GenBank (1014L10=KP763768, 1014L11 =KP763787, 1014L12=KP763792, 1014L13=KF697673, $1014 \mathrm{~L} 14=\mathrm{KF} 697679,1014 \mathrm{~F} 3=\mathrm{KP} 763782,1014 \mathrm{~F} 4=\mathrm{KF} 697680,1014 \mathrm{~F} 5=\mathrm{KF} 927156,1014 \mathrm{~F} 6=\mathrm{KP} 763803,1014 \mathrm{C2}=\mathrm{KF} 697683)$

sinensis heterozygote from Guiping of Guangxi [17]. This result is questionable and needs reconfirmation, because $1014 \mathrm{~F} / \mathrm{W}$ is not the only possibility encoded by the individual genotype $\mathrm{T}(\mathrm{T} / \mathrm{G})(\mathrm{T} / \mathrm{G})$. In this and another study [33], sequencing of cloned fragment with the $\mathrm{T}(\mathrm{T} / \mathrm{G})(\mathrm{T} / \mathrm{G})$ template resolved into two alleles, TTG and TGT, encoding 1014L/C rather than 1014F/W.

Differential $k d r$ distribution patterns were observed in the nine examined An. sinensis populations from Guangxi. Overall, haplotypes carrying 1014C mutation are widespread and present in high frequencies in the northeast, while 1014S is rare and distributed in the west (Table 1; Fig. 2). Notably, four of the nine populations with $k d r$ allele frequencies higher than $50 \%$ were located in the northeast of Guangxi, while only $1014 \mathrm{~S}$ in a frequency less than $5 \%$ was detected in the two western populations. Geographically, the frequency of $k d r$ mutations decreased towards south and west from northeast. This pattern is in keeping with previous observations that a high $k d r$ mutation frequency was detected in the central China which is located northeast to Guangxi [19, 34, 40], and $k d r$ alleles were less prevalent in Yunnan and Hainan which are in the west and south to Guangxi respectively $[16,18,19,34,40]$.

Considering the fact that haplotype $1014 \mathrm{C} 1$ and 1014F1, detected in samples of this study, are also distributed in central China (Table 1), it is possible that migration of An. sinensis population from central China through Hunan and Guizhou, may contribute to the occurrence of $1014 \mathrm{C} 1$ and $1014 \mathrm{~F} 1$ in the northeast of Guangxi. The obvious distinction in the geographic distribution of each allele between populations in the west and in the northeast is likely a combined consequence of 
Table 2 Haplotypes of the AS-VGSC gene and their frequencies (\%) in nine An. sinensis populations from Guangxi, China

\begin{tabular}{|c|c|c|c|c|c|c|c|c|c|}
\hline Haplotype & BS & GG & $\mathrm{GL}$ & $\mathrm{HC}$ & $\mathrm{HZ}$ & $\mathrm{LZ}$ & NN & WZ & $\mathrm{YL}$ \\
\hline 1014L1 & 27.3 & & 10.5 & 33.3 & & 13 & 38.6 & 10.4 & 31.7 \\
\hline 1014L2 & 51.8 & 6.7 & 13.2 & 38.5 & 10.5 & 11.1 & 24.3 & 16.7 & 14.4 \\
\hline $1014 \mathrm{~L} 3$ & 9.1 & 3.3 & 10.5 & 3.1 & 2.6 & 9.3 & 17.1 & 4.2 & 8.7 \\
\hline 1014L4 & 0.9 & & 2.6 & & & 3.7 & 5.7 & & 1.9 \\
\hline 1014L5 & 1.8 & & 4.0 & & & 3.7 & 1.4 & 8.3 & 1.9 \\
\hline $1014 L 6$ & 0.9 & & 1.3 & 1.0 & & & 4.3 & & 4.8 \\
\hline 1014L7 & & & & & & & 1.4 & & \\
\hline 1014L8 & 4.6 & 10.0 & 7.9 & 12.5 & 7.9 & 3.7 & 2.9 & 27.1 & 22.1 \\
\hline 1014L9 & 0.9 & & & & & & & & 1.0 \\
\hline $1014 \mathrm{~F} 1$ & & 6.7 & 13.2 & 1.0 & 7.9 & 16.7 & & 2.1 & 2.9 \\
\hline $1014 F 2$ & & & 1.3 & & 2.7 & 1.8 & & & \\
\hline $1014 S 1$ & & & & 1.1 & & & 1.4 & 8.3 & \\
\hline 101452 & 0.9 & & & 2.1 & & & 2.9 & & 2.9 \\
\hline 101453 & 0.9 & & & 5.2 & & & & & 1.0 \\
\hline 101454 & 0.9 & & & 1.1 & & 1.8 & & 2.1 & 3.8 \\
\hline $1014 C 1$ & & 73.3 & 35.5 & 1.1 & 68.4 & 35.2 & & 20.8 & 2.9 \\
\hline Mutant & 2.7 & 80.0 & 50.0 & 11.6 & 79.0 & 55.5 & 4.3 & 33.3 & 13.5 \\
\hline Size $(2 N)$ & 110 & 30 & 76 & 96 & 38 & 54 & 70 & 48 & 104 \\
\hline
\end{tabular}

Mutant means $1014 \mathrm{C} 1,1014 \mathrm{~F} 1,1014 \mathrm{~F} 2,1014 \mathrm{~S} 1,1014 \mathrm{~S} 2,1014 \mathrm{~S} 3,1014 \mathrm{~S} 4$ in this study. The italics value represents the frequency of the most abundant haplotype in the corresponding population. BS, GG, GL, HC, HZ, LZ, NN, WZ and YL are the abbreviations of Baise, Guigang, Guilin, Hechi, Hezhou, Liuzhou, Nanning, Wuzhou and Yulin respectively

independent mutational events in different geographic locations and the geographical barriers limiting gene flow imposed by the mountainous landscapes of Guangxi. Theoretically, selective pressure may play a role in shaping the frequency of insecticide resistance-associated mutation in a population. However, it was not possible to assess the contribution of local selection of insecticides to the existing $k d r$ distribution pattern because application history of insecticides was not known for samples used in this study.

Previous studies have documented that the predominant $k d r$ allele is L1014 F [4, 12, 13, 16, 19, 33-35, 40], the high frequency of $1014 \mathrm{C} 1$ in the five An. sinensis populations in northeast of Guangxi (Fig. 3) is unexpected. 1014C was present in An. sinensis samples collected in Anhui, Guizhou, Henan, Hubei, Hunan, Jiangsu and Shandong of China [16, 19, 34, 40] and in Korea [33], and was also found in VGSC of other mosquito species, such as Culex pipiens pipiens from China [36] and Anopheles albimanus from Mexico and Nicaragu [37]. These observations indicate that $1014 \mathrm{C}$ is a conserved mutation in mosquitoes and widely distributed. Why $1014 \mathrm{C} 1$ is prevalent in the five populations remains to be investigated. One possibility is that $1014 \mathrm{C} 1$ may provide a better protection, or/and a lower fitness cost, than other $k d r$ alleles such as the classical 1014F.

The geographic distribution pattern and the genealogical analysis of $k d r$ haplotypes strongly suggests that $k d r$ mutations are not singly originated. For example, there are at least two independent mutation events giving rise to $1014 \mathrm{~F}$ or $1014 \mathrm{~S}$ haplotypes from a wild haplotype through a single mutational step (Table 1; Fig. 3). Multiple origins of resistance alleles via point mutations at the voltage-gated sodium channel gene have also been characterized in other insect species [38, 39]. Interestingly, $1014 \mathrm{C} 1$ and $1014 \mathrm{~F} 1$ is co-distributed in Guangxi and share the same intron (Tables 1,2 ). The network analysis indicates that only one mutational step is able to change 1014F1 to 1014C1 (Fig. 3). Based on these observations, it is hypothesized that $1014 \mathrm{C} 1$ may represent a $k d r$ allele evolved via a sequential progression from 1014F1 and perhaps has gradually replaced 1014F1.

\section{Conclusions}

Sixteen AS-VGSC haplotypes were identified in An. sinensis from Guangxi, suggesting diverse genetic mutations in the $A S-V G S C$ gene. Three types of $k d r$ mutations (1014F, 1014S and 1014C) were detected in Guangxi. Genealogical analysis suggests multiple origins of $k d r$ mutations in An. sinensis. Although a direct link between $k d r$ mutations and level of insecticide resistance has not been established due to the unavailability of susceptibility data for the samples used in this study, the high frequency of $k d r$ mutation ( $>50 \%$ ) in Guigang, Guilin, Hezhou and Liuzhou indicates a risk of resistance to pyrethroids and DDT in An. sinensis in these areas. The 


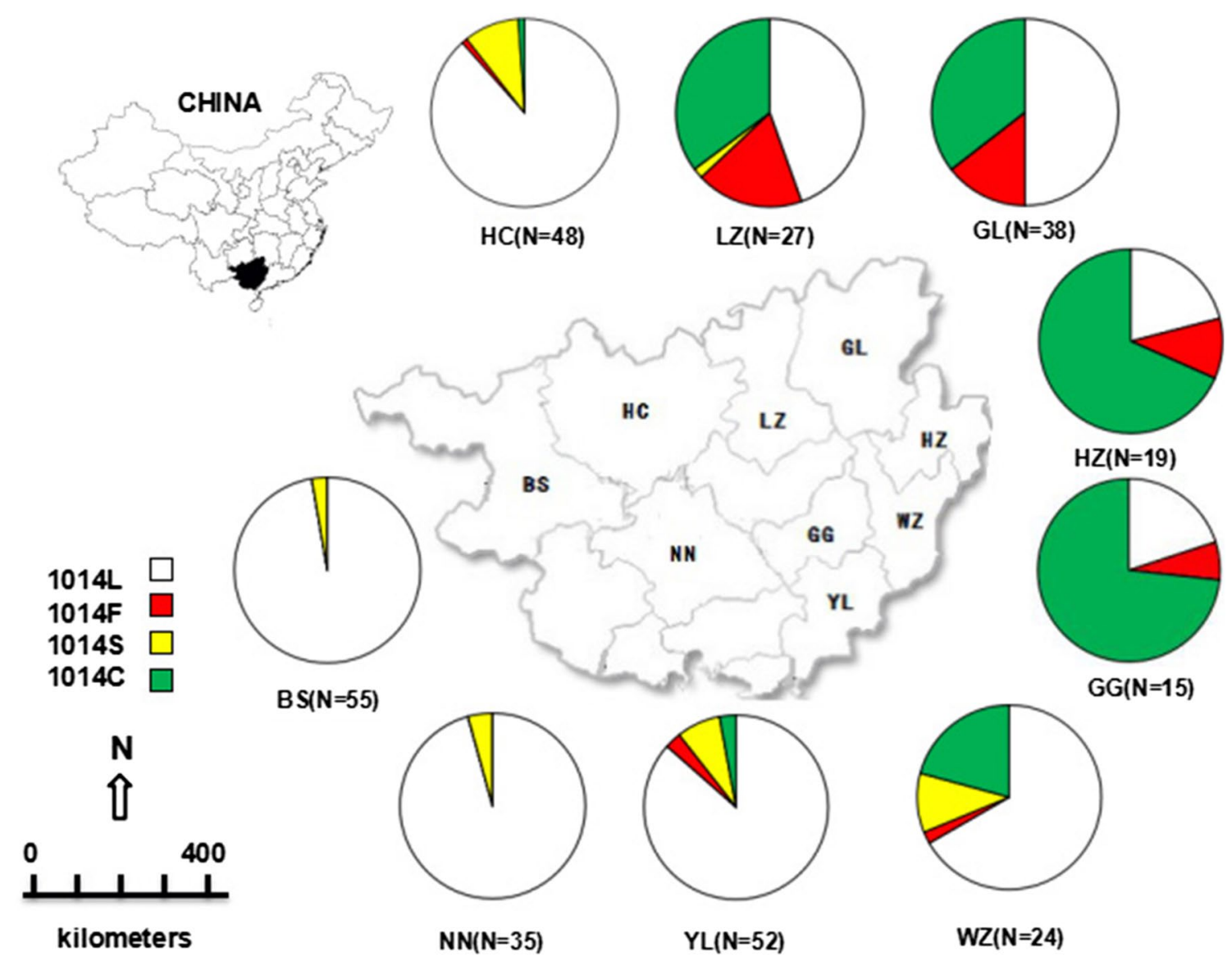

Fig. 2 Distribution of haplotypes in Anopheles sinensis in Guangxi. BS, HC, HZ, GG, GL, LZ, NN, WZ and YL are the abbreviations for Baise, Hechi, Hezhou, Guigang, Guilin, Liuzhou, Nanning, Wuzhou and Yulin respectively

Table 3 Frequency of VGSC genotypes in nine An. sinensis populations from Guangxi China, grouped according to the amino acid at position 1014 of VGSC

\begin{tabular}{|c|c|c|c|c|c|c|c|c|c|}
\hline Genotype & BS & GG & GL & $\mathrm{HC}$ & $\mathrm{HZ}$ & LZ & NN & WZ & $\mathrm{YL}$ \\
\hline $1014 \mathrm{~L} / \mathrm{L}$ & 94.6 & 6.7 & 29.1 & 77.0 & 5.3 & 18.5 & 91.3 & 41.6 & 73.4 \\
\hline $1014 \mathrm{~L} / \mathrm{F}$ & & 6.7 & 15.7 & 2.1 & & 25.9 & & 4.2 & 5.7 \\
\hline $1014 \mathrm{~L} / \mathrm{S}$ & 5.4 & & & 18.8 & & 3.7 & 8.7 & 20.9 & 15.2 \\
\hline $1014 \mathrm{~L} / \mathrm{C}$ & & 20.0 & 26.3 & 2.1 & 31.6 & 22.2 & & 25.0 & 5.7 \\
\hline $1014 \mathrm{~F} / \mathrm{F}$ & & & & & & 3.7 & & & \\
\hline $1014 \mathrm{C} / \mathrm{C}$ & & 60.0 & 15.8 & & 42.1 & 22.3 & & 8.3 & \\
\hline $1014 \mathrm{~F} / \mathrm{C}$ & & 6.7 & 13.1 & & 21.0 & 3.7 & & & \\
\hline Size $(N)$ & 55 & 15 & 38 & 48 & 19 & 27 & 35 & 24 & 52 \\
\hline
\end{tabular}

The italics value represents the frequency of the most abundant genotype in the corresponding population. BS, GG, GL, HC, HZ, LZ, NN, WZ and YL are the abbreviations of Baise, Guigang, Guilin, Hechi, Hezhou, Liuzhou, Nanning, Wuzhou and Yulin 


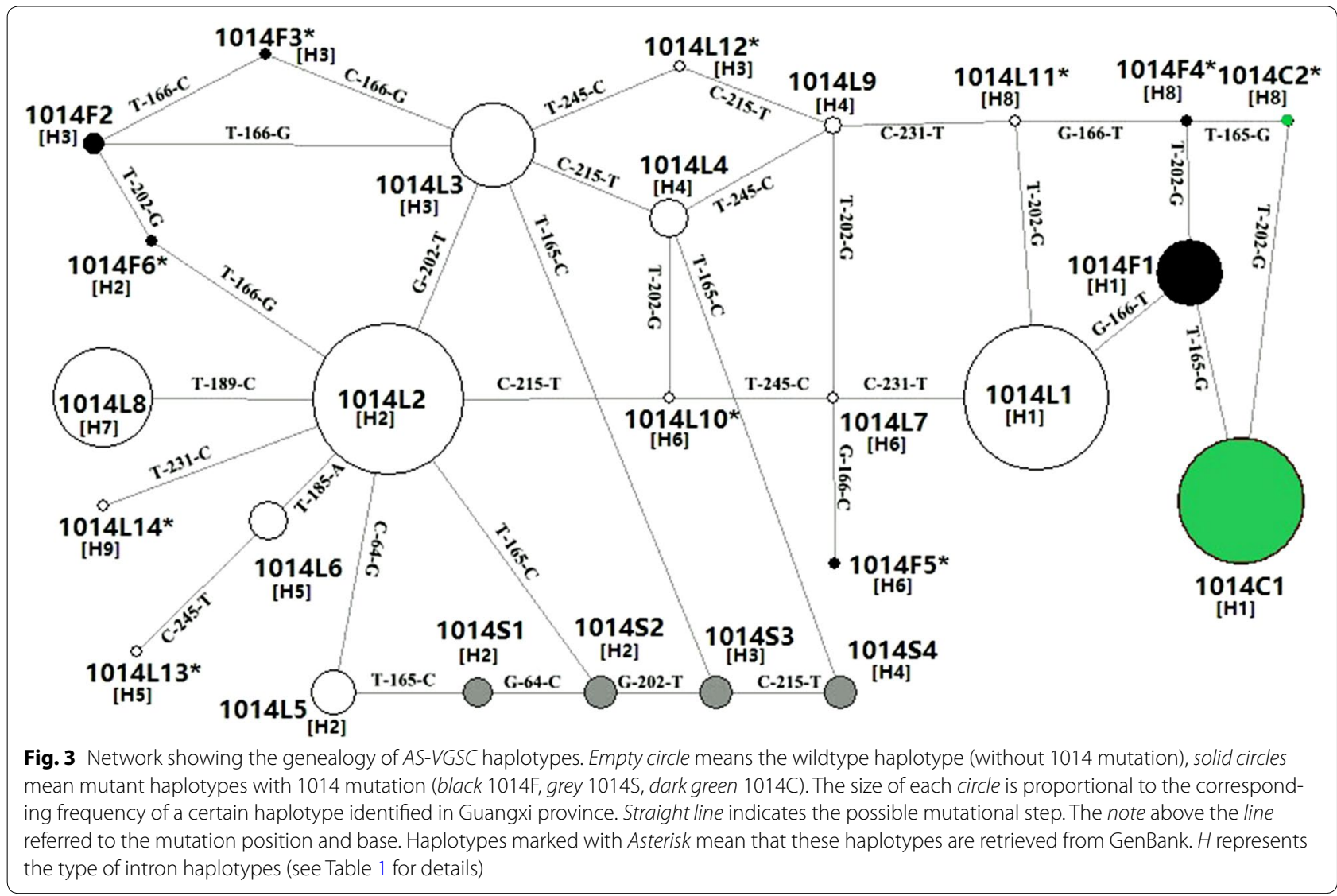

heterogeneities in the geographic distribution and multiple origins of $k d r$ alleles highlight the need of a locationcustomized strategy for monitoring and management of insecticide resistance.

\section{Additional files}

Additional file 1. Brief description of Anopheles sinensis collection sites in Guangxi.

Additional file 2. Genotypes of the AS-VGSC gene and their frequency in nine An. sinensis populations from Guangxi, China.

\section{Abbreviations}

VGSC: voltage-gated sodium channel; kdr: knockdown resistance; DDT: dichlorodiphenyltrichloroethane; ITNs: insecticide-treated bed nets; IRS: indoor residual spray.

\section{Authors' contributions}

$X H Q, C Y$ and $X Y F$ conceived the study. CY performed the experiment and XYF identified the species. $\mathrm{CY}, \mathrm{ZSH}$ and $\mathrm{XHQ}$ analyzed the data. $\mathrm{XHQ}, \mathrm{CY}, \mathrm{ZSH}$ and XYF wrote the paper. XYF and ML contributed to sample collection. All authors read and approved the final manuscript.

\section{Author details}

1 State Key Laboratory of Integrated Management of Pest Insects and Rodents, Institute of Zoology, Chinese Academy of Sciences, Beijing 100101, China.

${ }^{2}$ Guangxi Zhuang Autonomous Region Centre for Diseases Control and Prevention, Nanning 530028, China. ${ }^{3}$ University of Chinese Academy of Sciences, Beijing 100049, China.

\section{Acknowledgements}

The authors thank the reviewers for their constructive comments and suggestions

\section{Competing interests}

The authors declare that they have no competing interests.

\section{Availability of data and matarials}

All the datasets are presented in the main paper.

\section{Funding}

This work was supported by Grants No. 2012CB114103 and 2012ZX10004219. The funders had no role in the study design, data collection, analysis, decision to publish or preparation of the manuscript.

Received: 11 May 2016 Accepted: 30 July 2016

Published online: 15 August 2016

\section{References}

1. Enayati A, Hemingway J. Malaria management: past, present, and future. Annu Rev Entomol. 2010:55:569-91.

2. Lu B. Integrated mosquito management Second edition. 2nd ed. Beijing: Science Press; 1999.

3. Wang DQ, Xia ZG, Zhou SS, Zhou XN, Wang RB, Zhang QF. A potential threat to malaria elimination: extensive deltamethrin and DDT resistance to Anopheles sinensis from the malaria-endemic areas in China. Malar J. 2013;12:164. 
4. Zhang HW, Liu Y, Hu T, Zhou RM, Chen JS, Qian D, et al. Knockdown resistance of Anopheles sinensis in Henan province China. Malar J. 2015;14:137.

5. Dong K, Du Y, Rinkevich F, Nomura Y, Xu P, Wang L, et al. Molecular biology of insect sodium channels and pyrethroid resistance. Insect Biochem Mol Biol. 2014:50:1-17.

6. Silva APB, Santos JMM, Martins AJ. Mutations in the voltage-gated sodium channel gene of anophelines and their association with resistance to pyrethroids - a review. Parasit Vectors. 2014;7:450.

7. Brooke BD. kdr: can a single mutation produce an entire insecticide resistance phenotype? Trans R Soc Trop Med Hyg. 2008;102:524-5.

8. Donnelly MJ, Corbel V, Weetman D, Wilding CS, Williamson MS, Black WCT. Does $k d r$ genotype predict insecticide-resistance phenotype in mosquitoes? Trends Parasitol. 2009;25:213-9.

9. Burton MJ, Melloe IR, Duce IR, Davies TGE, Field LM, Williamson MS. Differential resistance of insect sodium channels with $k d r$ mutations to deltamethrin, permethrin and DDT. Insect Biochem Mol Biol. 2011:41:723-32

10. Du YZ, Nomura Y, Satar G, Hu ZN, Nauen R, He SY, et al. Molecular evidence for dual pyrethroid-receptor sites on a mosquito sodium channel. Proc Natl Acad Sci USA. 2013;110:11785-90.

11. Smith TJ, Lee SH, Ingles PJ, Knipple DC, Soderlund DM. The L1014F point mutation in the house fly VssCl sodium channel confers knockdown resistance to pyrethroids. Insect Biochem Mol Biol. 1997;27:807-12.

12. Tan JG, Liu ZQ, Nomura Y, Goldin AL, Dong K. Alternative splicing of and an insect sodium channel gene generates pharmacologically distinct sodium channels. J Neurosci. 2002;22:5300-9.

13. Tan JG, Liu ZQ, Wang RW, Huang ZY, Chen AC, Gurevitz M, et al. Identification of amino acid residues in the insect sodium channel critical for pyrethroid binding. Mol Pharmacol. 2005;67:513-22.

14. Usherwood PNR, Vais H, Khambay BPS, Davies TGE, Williamson MS Sensitivity of the Drosophila para sodium channel to DDT is not lowered by the super-kdr mutation M918T on the IIS4-S5 linker that profoundly reduces sensitivity to permethrin and deltamethrin. FEBS Lett. 2005;579:6317-25.

15. Vais H, Williamson MS, Goodson SJ, Devonshire AL, Warmke JW, Usherwood PN, et al. Activation of Drosophila sodium channels promotes modification by deltamethrin-Reductions in affinity caused by knockdown resistance mutations. J Gen Physiol. 2000;11:305-18.

16. Zhong D, Chang X, Zhou G, He Z, Fu F, Yan Z, et al. Relationship between knockdown resistance, metabolic detoxification and organismal resistance to pyrethroids in Anopheles sinensis. PLOS ONE. 2013;8:e55475.

17. Tan WL, Li CX, Wang ZM, Liu MD, Dong YD, Feng XY, et al. First Detection of multiple knockdown resistance $(k d r)$ like mutations in voltage-gated sodium channel using three new genotyping methods in Anopheles sinensis from Guangxi Province, China. J Med Entomol. 2012;49:1012-20.

18. Qin Q, Li YJ, Zhong DB, Zhou N, Chang XL, Li C, et al. Insecticide resistance of Anopheles sinensis in Hainan Island, a malaria-endemic area of China. Parasit Vectors. 2014;7:92.

19. Wang Y, Yu WQ, Shi H, Yang ZZ, Xu JN, Ma YJ, et al. Historical survey of the $k d r$ mutations in the populations of Anopheles sinensis in China in 1996-2014. Malar J. 2015:14:120.

20. Li J, Wei S, Yang Y, Li J. Analysis of the prevalence of imported malaria in Guangxi from 2012 to 2013. J Pathog Biology. 2015;10:180-3.

21. $L i J H, L i J$, Qin $Y X$, Guo CK, Huang YM, Lin Z. Appraisal of the effect and measures on control malaria for 60 years in Guangxi. J Trop Med. 2014;14:361-4.

22. Li J, Yang Y, Zhang W, Wei S, Lin K. Mid-term assessment report of malaria elimination action plan in Guangxi. China Tropical Medicine. 2016;16:305-10

23. Lu G, Zhou S, Horstick O, Wang X, Liu Y, Müller O. Malaria outbreaks in China (1990-2013): a systematic review. Malar J. 2014;13:269.
24. Lin K, Huang Y, Li J, Mao W, Li J, Xun Y, et al. Malaria vector Anopheles investigation analysis after malaria was basically eliminated in Guangxi. J Med Pest Control. 2014;30:963-6.

25. Guo C, Li J, Qin Y. Investigation on geographical distribution, ecological feature and malaria transmission of Anopheles in Guangxi. Chin J Vector Biol Control. 2007;18:112-5.

26. Lu B, Wu H. Classification and identification of important medical insects of China. 1st ed. China: Henan Science and Technology Publishing House: 2003.

27. Joshi D, Park MH, Saeung A, Choochote W, Min GS. Multiplex assay to identify Korean vectors of malaria. Mol Ecol Resour. 2010;10:748-50.

28. Feng $X$, Yang C, Yang Y, Li J, Lin K, Li M, Qiu X. Distribution and frequency of G119S mutation in acel gene within Anopheles sinensis populations from Guangxi, China. Malar J. 2015;14:470

29. Rinkevich FD, Zhang L, Hamm RL, Brady SG, Lazzaro BP, Scott JG. Frequencies of the pyrethroid resistance alleles of VssC1 and CYP6D1 in house flies from the eastern United States. Insect Mol Biol. 2006;15:157-67.

30. Bandelt $H$, Forster $P$, Rohl A. Median-joining networks for inferring intraspecific phylogenies. Mol Biol Evol. 1999;16:37-48.

31. Clement M, Posada D, Crandall K. TCS: a computer program to estimate gene genealogies. Mol Ecol. 2000;9:1657-9.

32. Kang $\mathrm{S}$, Jung J, Lee $\mathrm{S}$, Hwang $\mathrm{H}$, Kim W. The polymorphism and the geographical distribution of the knockdown resistance (kdr) of Anopheles sinensis in the Republic of Korea. Malar J. 2012;11:151-8.

33. Kim H, Baek JH, Lee WJ, Lee SH. Frequency detection of pyrethroid resistance allele in Anopheles sinensis populations by realtime PCR amplification of specific allele (rtPASA). Pestic Biochem Phys. 2007:87:54-61.

34. Chang XL, Zhong DB, Fang Q, Hartsel J, Zhou GF, Shi LN, et al. Multiple resistances and complex mechanisms of Anopheles sinensis mosquito: a major obstacle to mosquito-borne diseases control and elimination in China. PLoS Neglect Trop Dis. 2014;8:e2889.

35. Bai L, Zhu GD, Zhou HY, Tang JX, Li JL, Xu S, et al. Development and application of an AllGlo probe-based qPCR assay for detecting knockdown resistance ( $k d r$ ) mutations in Anopheles sinensis. Malar J. 2014;13:379.

36. Wang ZM, Li CX, Xing D, Yu YH, Liu N, Xue RD, et al. Detection and widespread distribution of sodium channel alleles characteristic of insecticide resistance in Culex pipiens complex mosquitoes in China. Med Vet Entomol. 2012;26:228-32.

37. Lol JC, Castellanos ME, Liebman KA, Lenhart A, Pennington PM, Padilla NR. Molecular evidence for historical presence of knock-down resistance in Anopheles albimanus, a key malaria vector in Latin America. Parasit Vectors. 2013:6:268.

38. Rinkevich FD, Hedtke SM, Leichter CA, Harris SA, Su C, Brady SG, et al. Multiple origins of $k d r$-type resistance in the house fly. Musca domestica. PLoS One. 2012;7:e52761.

39. Pinto J, Lynd A, Vicente JL, Santolamazza F, Randle NP, Gentile G, et al. Multiple origins of knockdown resistance mutations in the afrotropical mosquito vector Anopheles gambiae. PLoS ONE. 2007;2:e1243.

40. Chang X, Zhong D, Lo E, Fang Q, Bonizzoni M, Wang X, et al. Landscape genetic structure and evolutionary genetics of insecticide resistance gene mutations in Anopheles sinensis. Parasit Vectors. 2016;9:228.

\section{Submit your next manuscript to BioMed Central and we will help you at every step:}

- We accept pre-submission inquiries

- Our selector tool helps you to find the most relevant journal

- We provide round the clock customer support

- Convenient online submission

- Thorough peer review

- Inclusion in PubMed and all major indexing services

- Maximum visibility for your research

Submit your manuscript at www.biomedcentral com/submit
BioMed Central 